\title{
Discriminating Among the Log-Normal, Weibull and Generalized Exponential Distributions
}

\author{
Arabin Kumar DeY ${ }^{\dagger} \&$ Debasis $\mathrm{Kundu}^{\dagger}$
}

\begin{abstract}
In this paper we consider the model selection/ discrimination among the three important lifetime distributions. All these three distributions have been used quite effectively to analyze lifetime data in the reliability analysis. We study the probability of correct selection using the maximized likelihood method, as it has been used in the literature. We further compute the asymptotic probability of correct selection and compare the theoretical and simulation results for different sample sizes and for different model parameters. The results have been extended for Type-I censored data also. The theoretical and simulation results match quite well. Two real data sets have been analyzed for illustrative purposes. We also suggest a method to determine the minimum sample size required to discriminate among the three distributions for a given probability of correct selection and a user specified protection level.
\end{abstract}

KEYWORDS: Model selection; likelihood ratio test; probability of correct selection; KolmogorovSmirnov distance; bivariate normal distribution.

$\dagger$ Department of Mathematics and Statistics, Indian Institute of Technology Kanpur, Kanpur, Pin 208016, INDIA.

Corresponding Author: Debasis Kundu, Phone no. 91-512-2597141, Fax No. 91-5122597500, e-mail: kundu@iitk.ac.in. 


\section{INTRODUCTION}

Log-normal, Weibull and generalized exponential distributions have been used quite effectively in analyzing positively skewed data, which play important roles in the reliability analysis. Often it is assumed that the data are coming from a specific parametric family and the rest of the analysis are done based on that model assumption. But often choosing a particular model is quite difficult and the effect due to model mis-specification can be quite severe.

Suppose $\left\{x_{1}, \ldots, x_{n}\right\}$ is a univariate lifetime data, i.e. each $x_{i}>0$, and the preliminary descriptive data analysis indicates that it is coming from a positively skewed distribution function. Therefore, any of the three distributions can be used to model the data and all of them may fit the data very well. It is observed that for certain ranges of the parameter values the corresponding probability density functions (PDFs) or the cumulative distribution functions (CDFs) are very close to each other but some of the other characteristics can be quite different. Before explaining this with an example, we introduce the following notation. For $\beta>0, \theta>0, \sigma>0, \eta>0, \alpha>0, \lambda>0, W E(\beta, \theta), L N(\sigma, \eta)$ and $G E(\alpha, \lambda)$ denote Weibull, log-normal and generalized exponential distributions with shape and scale parameters as $(\beta, \theta),(\sigma, \eta)$ and $(\alpha, \lambda)$ respectively. The corresponding PDFs respectively are

WEIBULL:

$$
f_{W E}(x ; \alpha, \theta)=\beta \theta^{\beta} x^{\beta-1} e^{-(x \theta)^{\beta}} ; \quad x>0,
$$

LOG-NORMAL:

$$
f_{L N}(x ; \sigma, \eta)=\frac{1}{\sqrt{2 \pi} x \sigma} e^{-\frac{(\ln x-\ln \eta)^{2}}{2 \sigma^{2}}} ; \quad x>0,
$$

Generalized Exponential:

$$
f_{G E}(x ; \alpha, \lambda)=\alpha \lambda\left(1-e^{-\lambda x}\right)^{\alpha-1} e^{-\lambda x} ; \quad x>0 .
$$


Now we discuss briefly the necessity of choosing the correct model if all the models fit the data reasonably well. Cox [5] first discussed the effect of choosing the wrong model. Wiens [26] demonstrated it nicely by a real data example and recently Pascual [18] also provided the effect of mis-specification on the maximum likelihood estimates between two distribution functions.

Consider, the CDFs of $G E(3,1), W E(1.67,0.48)$ and $L N(0.45,1.50)$ in Figure 1. From the Figure 1 the closeness of the three CDFs can be easily visualized. If the data are coming

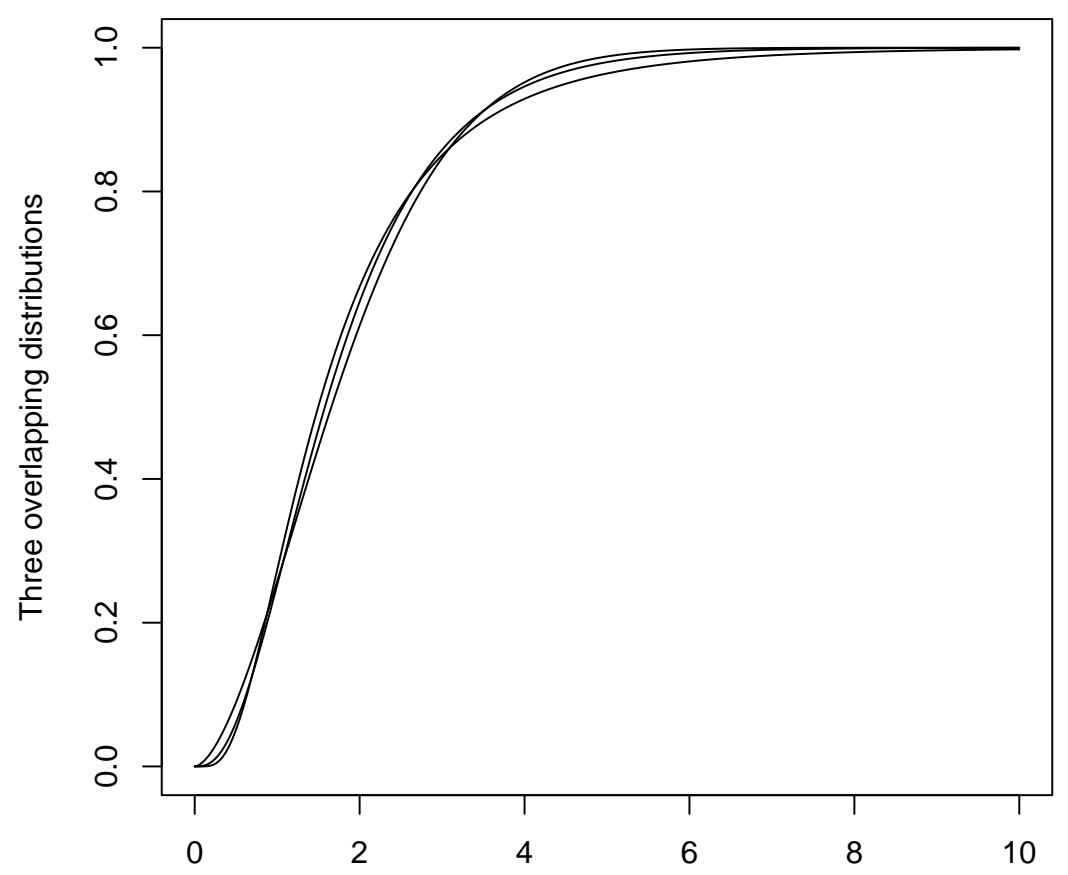

Figure 1: The CDFs of $G E(3,1), W E(1.67,0.48)$ and $L N(0.45,1.50)$

from one of the three distribution functions, it can be easily modeled by any of the other two distribution functions also. Therefore, the natural questions are how to choose the correct model and how reliable is this procedure? The problem of choosing the exact distribution 
becomes more difficult if the sample size is not very large or the data are censored. If the data are coming from $G E(3,1)$, then the probability of correct selection (PCS) based on Monte Carlo simulations using the maximized likelihood method (the details will be explained in Section 2), is as follows:

\begin{tabular}{|l|l|l|l|l|l|l|}
\hline Sample Size & 20 & 40 & 60 & 80 & 100 & 500 \\
\hline PCS & 0.265 & 0.428 & 0.523 & 0.599 & 0.659 & 0.976 \\
\hline
\end{tabular}

It is important to observe that the PCS can be as small as only 0.27 when the sample size is 20. On the other hand the effect of mis-specification can be quite severe. Let us look at the hazard functions or the mean residual life of the three distribution functions in Figures 2 and 3 respectively. The lower 5-th percentile points of those three distributions are $0.5273(\mathrm{GE}) \& 0.3518(\mathrm{WE}) \& 0.7155(\mathrm{LN})$ respectively. It is clear that although the three CDFs are quite close to each other, but some of the other characteristics of the distribution functions can be quite different. The problem due to mis-specification may be observed in other cases also, for example in variable sampling plan, see Schneider [21], constructing confidence bounds, see for example Keats, Nahar and Korbell [11], or even in transformation, see for example Xie, Yang and Gaudoin [27].

The problem of choosing the correct distribution is an old problem in the statistical literature. The problem of testing whether some given observations follow one of the two possible distribution functions has been attempted by many researchers. Cox [5,6] first considered this problem in general for discriminating between two non-nested models and then Atkinson [1], Chen [4], Chambers and Cox [3] also made significant contributions in this problem. Due to increasing applications of the lifetime distributions, special attention has been paid in discriminating between the log-normal and Weibull distributions (Dumonceaux and Antle [7], Pereira [19], Chen [4], Quesenberry and Kent [23], Kundu and Manglick [13], 


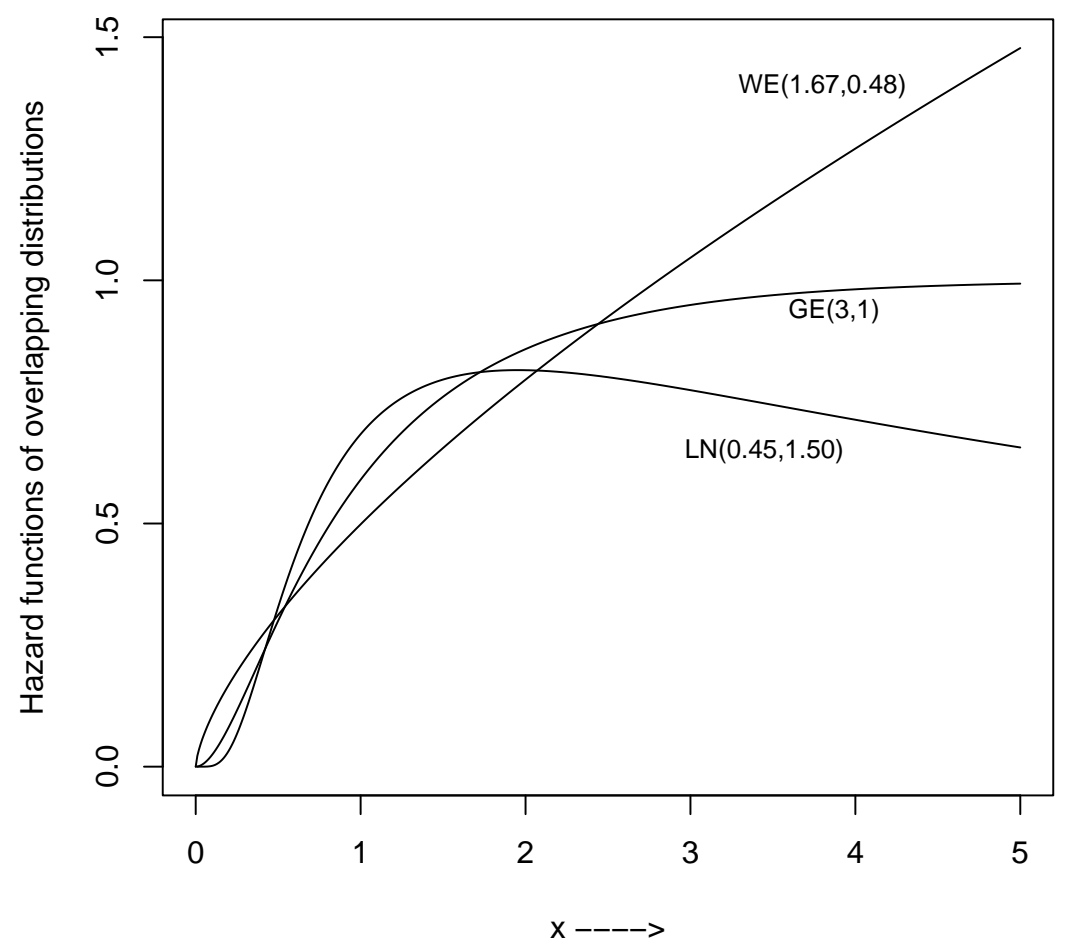

Figure 2: The hazard functions of $G E(3,1), W E(1.67,0.48)$ and $L N(0.45,1.50)$

Pascual [18]), the gamma and Weibull distributions (Bain and Englehardt [2], Fearn and Nebenzahl [8]), the gamma and log-normal distributions (Kundu and Manglick [14]), the log-normal and generalized exponential distributions (Kundu, Gupta and Manglick; [12]), the Weibull and generalized exponential distributions (Gupta and Kundu [9]). Although, significant amount of work has been done on discriminating between two distributions, but not much work has been done except the work of Marshal, Meza and Olkin [17], Kappenman [10] and Taylor and Jakeman [22] in discriminating more than two distributions. Moreover no work has been done when the data are censored, which is very common in the reliability analysis.

The aim of this paper is two fold. First of all we consider the discrimination procedure of 


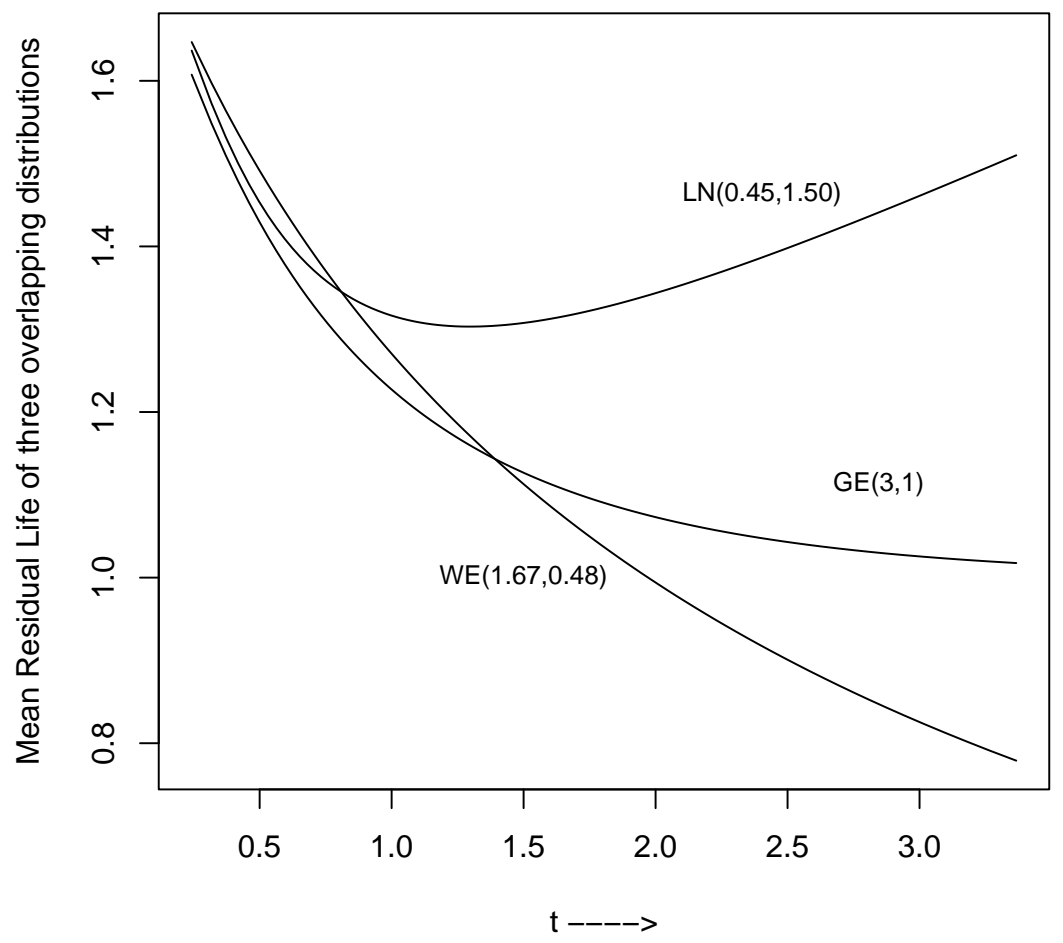

Figure 3: The mean residual lives of $G E(3,1), W E(1.67,0.48)$ and $L N(0.45,1.50)$

the three well known distribution functions, which have been used quite extensively in the reliability analysis. We use the maximized likelihoods method in choosing the best possible distribution. We obtain the asymptotic distributions of the maximized likelihood functions. Since the theoretical finite sample results are not available, we compute the probability of correct selection for different sample sizes using the asymptotic distributions. We compare the finite sample and asymptotic results, by extensive computer simulations. It is observed that the theoretical and the experimental results match quite well even for moderate sample sizes. Results have been extended when the data are Type-I censored. We provide the analysis of two data sets for illustrative purposes. Secondly, we also provide the minimum sample size required to obtain a given probability of correct selection for a user specified protection level in choosing the correct distribution. 
The rest of the paper is organized as follows. In section 2, we provide the discrimination procedure. The asymptotic probability of correct selections are provided in section 3. Comparison of the simulation and experimental results are presented in section 4. The results of Type-I censored data are provided in section 5. For illustrative purposes two data analysis results are presented in section 6. Sample size determination is provided in section 7 and finally the conclusion appears in section 7 .

\section{Discrimination Procedure}

In this section we describe the discrimination procedure on the basis of a random sample $X=\left\{x_{1}, \ldots, x_{n}\right\}$. It is assumed that the data have been generated from one of the three distributions, namely $W E(\beta, \theta), L N(\sigma, \eta)$ or $G E(\alpha, \lambda)$ and the corresponding likelihood functions respectively are

$$
L_{W E}(\beta, \theta)=\prod_{i=1}^{n} f_{W E}\left(x_{i} ; \beta, \theta\right), L_{L N}(\sigma, \eta)=\prod_{i=1}^{n} f_{L N}\left(x_{i} ; \sigma, \eta\right), L_{G E}(\alpha, \lambda)=\prod_{i=1}^{n} f_{G E}\left(x_{i} ; \alpha, \lambda\right) .
$$

If $\widehat{\beta}, \widehat{\theta}, \widehat{\sigma}, \widehat{\eta}, \widehat{\alpha}, \widehat{\lambda}$ are the maximum likelihood estimators of the corresponding parameters, then choose that particular distribution with the largest maximized likelihood value. Now we would like to study the probability of correct selection (PCS) in each case. For example, if the data are actually coming from $W E(\beta, \theta)$, then the probability that

$$
L_{W E}(\widehat{\beta}, \widehat{\theta})>\max \left\{L_{L N}(\widehat{\sigma}, \widehat{\eta}), L_{G E}(\widehat{\alpha}, \widehat{\lambda})\right\}
$$

and similarly for the other cases also. We consider the three cases separately. Let us consider the following statistics;

$$
L_{1}=\frac{L_{W E}(\widehat{\beta}, \widehat{\theta})}{L_{L N}(\widehat{\sigma}, \widehat{\eta})}, \quad L_{2}=\frac{L_{W E}(\widehat{\beta}, \widehat{\theta})}{L_{G E}(\widehat{\alpha}, \widehat{\lambda})} \quad L_{3}=\frac{L_{L N}(\widehat{\sigma}, \widehat{\eta})}{L_{G E}(\widehat{\alpha}, \widehat{\lambda})}
$$

and the corresponding logarithms are $T_{i}=\ln L_{i}$ for $i=1, \ldots, 3$. Therefore, the PCS when the data follow Weibull distribution is

$$
P\left(T_{1}>0, T_{2}>0 \mid \text { data follow Weibull }\right)=P_{W E}\left(T_{1}>0, T_{2}>0\right) \quad \text { (say) } .
$$


Similarly, in case of log-normal and generalized exponential distributions, the PCS can be written as

$$
P_{L N}\left(T_{3}>0, T_{1}<0\right)=P\left(T_{3}>0, T_{1}<0 \mid \text { data follow log-normal }\right),
$$

and

$$
P\left(T_{2}<0, T_{3}<0 \mid \text { data follow } \mathrm{GE}\right)=P_{G E}\left(T_{2}<0, T_{3}<0\right),
$$

respectively.

Now let us look at the expressions of the $T_{i}$ 's in terms of the corresponding MLEs.

$$
\begin{aligned}
& T_{1}=n\left[\ln \left(\widehat{\sigma} \widehat{\beta}(\widehat{\theta} \widehat{\eta})^{\widehat{\beta}} \sqrt{2 \pi}\right)-\frac{1}{2}\right], \\
& T_{2}=n\left[\frac{\widehat{\alpha}-1}{\widehat{\alpha}}+\hat{\lambda} \bar{X}+\widehat{\beta} \ln (\widehat{\theta} \tilde{X})-\ln \left(\frac{\widehat{\alpha} \widehat{\lambda} \tilde{X}}{\widehat{\beta}}\right)-1\right], \\
& T_{3}=n\left[\frac{\widehat{\alpha}-1}{\widehat{\alpha}}+\hat{\lambda} \bar{X}-\frac{1}{2}(1+\ln (2 \pi))-\ln (\widehat{\alpha} \hat{\lambda} \tilde{X} \widehat{\sigma})\right] .
\end{aligned}
$$

Here

$$
\bar{X}=\frac{1}{n} \sum_{i=1}^{n} X_{i}, \quad \tilde{X}=\left\{\prod_{i=1}^{n} X_{i}\right\}^{\frac{1}{n}}
$$

moreover

$$
\widehat{\eta}=\tilde{X}, \quad \widehat{\sigma}^{2}=\frac{1}{n} \sum_{i=1}^{n}\left[\ln X_{i}-\ln \widehat{\eta}\right]^{2}, \quad \widehat{\theta}=\left[\frac{n}{\sum_{i=1}^{n} X_{i}^{\widehat{\beta}}}\right]^{\frac{1}{\hat{\beta}}} \quad \widehat{\alpha}=-\frac{n}{\sum_{i=1}^{n} \ln \left(1-e^{-\widehat{\lambda} X_{i}}\right)} .
$$

\section{Asymptotic Distributions}

In this section first we compute the joint distributions of $\left\{T_{1}, T_{2}\right\},\left\{T_{1}, T_{3}\right\}$ and $\left\{T_{2}, T_{3}\right\}$ for three cases, and these will be used to compute the PCSs asymptotically. From now on we denote the almost sure convergence by a.s.. For any functions $g(\cdot)$ and $h(\cdot), E_{W E}(g(U))$, $V_{W E}(g(U)), \operatorname{Cov}_{W E}(g(U), h(U))$ denote the mean of $g(U)$, variance of $g(U)$ and covariance between $g(U)$ and $h(U)$ respectively, if $U$ follows $W E(\beta, \theta)$. Similarly, we define when $U$ 
follows $L N(\sigma, \eta)$ or $G E(\alpha, \lambda)$ and they should be clear from the context and notations. Now we provide the main results.

ThEOREM 1: Under the assumptions that the data are from $W E(\beta, \theta)$, then $\left(T_{1}, T_{2}\right)^{T}$ is asymptotically bivariate normally distributed with mean vector $\left(E_{W E}\left(T_{1}\right), E_{W E}\left(T_{2}\right)\right)^{T}$ and dispersion matrix

$$
\Sigma_{W E}=\left[\begin{array}{cc}
V_{W E}\left(T_{1}\right) & \operatorname{Cov}_{W E}\left(T_{1}, T_{2}\right) \\
\operatorname{Cov}_{W E}\left(T_{1}, T_{2}\right) & V_{W E}\left(T_{2}\right)
\end{array}\right]
$$

To prove Theorem 1, we need the following lemma.

LEMma 1: Under the assumption that the data are from $W E(\beta, \theta)$, we have the following results as $n \longrightarrow \infty$.

(a) $\widehat{\beta} \longrightarrow \beta \quad$ a.s, $\quad \widehat{\theta} \longrightarrow \theta \quad$ a.s., $\quad$ where

$$
E_{W E}\left[\ln f_{W E}(X ; \beta, \theta)\right]=\max _{\bar{\beta}, \bar{\theta}} E_{W E}\left[\ln f_{W E}(X ; \bar{\beta}, \bar{\theta})\right]
$$

(b) $\widehat{\sigma} \longrightarrow \sigma_{1}$, a.s, $\hat{\eta} \longrightarrow \eta_{1} \quad$ a.s., $\quad$ where

$$
E_{W E}\left[\ln f_{L N}\left(X ; \sigma_{1}, \eta_{1}\right)\right]=\max _{\sigma, \eta} E_{W E}\left[\ln f_{L N}(X ; \sigma, \eta)\right]
$$

(c) $\widehat{\alpha} \longrightarrow \alpha_{1}$, a.s, $\quad \widehat{\lambda} \longrightarrow \lambda_{1} \quad$ a.s., $\quad$ where

$$
E_{W E}\left[\ln f_{G E}\left(X ; \alpha_{1}, \lambda_{1}\right)\right]=\max _{\alpha, \lambda} E_{W E}\left[\ln f_{G E}(X ; \alpha, \lambda)\right]
$$

Note that $\sigma_{1}, \eta_{1}, \alpha_{1}$ and $\lambda_{1}$ may depend on $\beta$ and $\theta$ but we do not make it explicit for brevity. Now, let us denote

$$
T_{1}^{*}=\ln \left[\frac{L_{W E}(\beta, \theta)}{L_{L N}\left(\sigma_{1}, \eta_{1}\right)}\right], \quad T_{2}^{*}=\ln \left[\frac{L_{W E}(\beta, \theta)}{L_{G E}\left(\alpha_{1}, \lambda_{1}\right)}\right] .
$$

(d)

$$
\frac{1}{\sqrt{n}}\left[T_{1}-E_{W E}\left(T_{1}\right), \quad T_{2}-E_{W E}\left(T_{2}\right)\right]^{T} \stackrel{\text { a.e. }}{=} \frac{1}{\sqrt{n}}\left[T_{1}^{*}-E_{W E}\left(T_{1}^{*}\right), \quad T_{2}^{*}-E_{W E}\left(T_{2}^{*}\right)\right]^{T},
$$

here $\stackrel{\text { a.e.d }}{=}$ means asymptotically equivalent in distribution. 
Proof of Lemma 1: The proof of Lemma 1 is not difficult. It mainly follows using similar arguments of White ([24], Theorem 1) and therefore it is omitted.

Proof of Theorem 1: Using Central Limit Theorem, it immediately follows that $T_{1}^{*}$ and $T_{2}^{*}$ are asymptotically normally distributed. Moreover it easily follows that any linear combinations of $T_{1}^{*}$ and $T_{2}^{*}$ are also asymptotically normally distributed, therefore the bivariate normality result also immediately follows.

Along the same line it can be shown that both $\left(T_{1}, T_{3}\right)$ and $\left(T_{2}, T_{3}\right)$ are asymptotically bivariate normally distributed. The expressions of the elements of mean vectors and the dispersion matrices are provided in the Appendix A. Using the exact expressions of the different elements of the mean vectors and the covariance matrices, the asymptotic PCS can be obtained numerically by performing double integrations on the proper quadrant.

\section{Numerical Results}

In this section we perform some simulation experiments mainly to observe how the PCSs based on the asymptotic distributions work for different sample sizes and for different parameter values. All the computations are performed at the Indian Institute of Technology Kanpur using S-PLUS and C. The programs can be obtained from the authors on request.

We consider different parameter values and different sample sizes for three different cases. In all the cases we have taken the scale parameters to be one. We have mainly varied the shape parameter. For each parameter values and for fixed sample size $n$, we have generated a sample of size $n$, from a particular distribution function (Weibull, log-normal or GE) and then find the best fitted distribution based on the criterion given in section 2 . We repeat this process 10000 times and compute the percentage of times it chooses the correct distribution function. We also compute the asymptotic PCS based on the result provided in section 3. 
All the results are reported in Tables 1,2 and 3. The elements in the first row in each box represent the results based on Monte Carlo simulations and the numbers in bracket immediately below represent the results obtained by asymptotic results.

Table 1: The probability of correct selection based on Monte Carlo (MC) simulations and also based on asymptotic results (AR) when the data are from Weibull distribution.

\begin{tabular}{|c|c|c|c|c|c|c|c|}
\hline $\begin{array}{l}n \longrightarrow \\
\beta \downarrow\end{array}$ & & 20 & 40 & 60 & 80 & 100 & 500 \\
\hline 0.6 & $\begin{array}{l}\text { MC } \\
\text { AR }\end{array}$ & $\begin{array}{l}0.355 \\
(0.435)\end{array}$ & $\begin{array}{l}0.537 \\
(0.562)\end{array}$ & $\begin{array}{l}0.644 \\
(0.645)\end{array}$ & $\begin{array}{l}0.686 \\
(0.705)\end{array}$ & $\begin{array}{l}0.742 \\
(0.750)\end{array}$ & $\begin{array}{l}0.974 \\
(0.967)\end{array}$ \\
\hline 0.8 & $\begin{array}{l}\mathrm{MC} \\
\mathrm{AR}\end{array}$ & $\begin{array}{l}0.338 \\
(0.362)\end{array}$ & $\begin{array}{l}0.444 \\
(0.463)\end{array}$ & $\begin{array}{l}0.524 \\
(0.528)\end{array}$ & $\begin{array}{l}0.527 \\
(0.575)\end{array}$ & $\begin{array}{l}0.573 \\
(0.610)\end{array}$ & $\begin{array}{l}0.801 \\
(0.813)\end{array}$ \\
\hline 1.0 & $\begin{array}{l}\mathrm{MC} \\
\mathrm{AR}\end{array}$ & $\begin{array}{l}0.424 \\
(0.420)\end{array}$ & $\begin{array}{l}0.461 \\
(0.475)\end{array}$ & $\begin{array}{l}0.472 \\
(0.475)\end{array}$ & $\begin{array}{l}0.482 \\
(0.487)\end{array}$ & $\begin{array}{l}0.485 \\
(0.495)\end{array}$ & $\begin{array}{l}0.499 \\
(0.519)\end{array}$ \\
\hline 1.2 & $\begin{array}{l}\mathrm{MC} \\
\mathrm{AR}\end{array}$ & $\begin{array}{l}0.544 \\
(0.546)\end{array}$ & $\begin{array}{l}0.586 \\
(0.581)\end{array}$ & $\begin{array}{l}0.613 \\
(0.603)\end{array}$ & $\begin{array}{l}0.660 \\
(0.620)\end{array}$ & $\begin{array}{l}0.677 \\
(0.635)\end{array}$ & $\begin{array}{l}0.796 \\
(0.781)\end{array}$ \\
\hline 1.4 & $\begin{array}{l}\mathrm{MC} \\
\mathrm{AR}\end{array}$ & $\begin{array}{l}0.620 \\
(0.594)\end{array}$ & $\begin{array}{l}0.672 \\
(0.650)\end{array}$ & $\begin{array}{l}0.700 \\
(0.687)\end{array}$ & $\begin{array}{l}0.751 \\
(0.715)\end{array}$ & $\begin{array}{l}0.772 \\
(0.739)\end{array}$ & $\begin{array}{l}0.932 \\
(0.925)\end{array}$ \\
\hline 1.6 & $\begin{array}{l}\mathrm{MC} \\
\mathrm{AR}\end{array}$ & $\begin{array}{l}0.658 \\
(0.634)\end{array}$ & $\begin{array}{l}0.724 \\
(0.704)\end{array}$ & $\begin{array}{l}0.757 \\
(0.751)\end{array}$ & $\begin{array}{l}0.812 \\
(0.786)\end{array}$ & $\begin{array}{l}0.835 \\
(0.813)\end{array}$ & $\begin{array}{l}0.978 \\
(0.977)\end{array}$ \\
\hline 1.8 & $\begin{array}{l}\mathrm{MC} \\
\mathrm{AR}\end{array}$ & $\begin{array}{l}0.686 \\
(0.666)\end{array}$ & $\begin{array}{l}0.765 \\
(0.746)\end{array}$ & $\begin{array}{l}0.801 \\
(0.798)\end{array}$ & $\begin{array}{l}0.854 \\
(0.836)\end{array}$ & $\begin{array}{l}0.880 \\
(0.864)\end{array}$ & $\begin{array}{l}0.994 \\
(0.993)\end{array}$ \\
\hline 2.0 & $\begin{array}{l}\text { MC } \\
\text { AR }\end{array}$ & $\begin{array}{l}0.707 \\
(0.691)\end{array}$ & $\begin{array}{l}0.792 \\
(0.778)\end{array}$ & $\begin{array}{l}0.833 \\
(0.832)\end{array}$ & $\begin{array}{l}0.890 \\
(0.871)\end{array}$ & $\begin{array}{l}0.913 \\
(0.899)\end{array}$ & $\begin{array}{l}0.998 \\
(0.998)\end{array}$ \\
\hline
\end{tabular}

Some of the points are quite clear from the experiments. As the sample size increases the PCS increases as expected. The asymptotic results match reasonably well in all cases considered even when the sample size is not very large. When the data are drawn from the Weibull distribution, then the PCSs increase as the shape parameter increases from 1 for all sample sizes. The PCSs remain more or less constant as the shape parameter 
Table 2: The probability of correct selection based on Monte Carlo (MC) simulations and also based on asymptotic results (AR) when the data are from log-normal distribution.

\begin{tabular}{|l|l|l|l|l|l|l|l|}
\hline$n \longrightarrow$ & & 20 & 40 & 60 & 80 & 100 & 500 \\
$\sigma \downarrow$ & & & & & & & \\
\hline 0.5 & $\mathrm{MC}$ & 0.583 & 0.642 & 0.662 & 0.677 & 0.698 & 0.861 \\
& $\mathrm{AR}$ & $(0.572)$ & $(0.612)$ & $(0.638)$ & $(0.659)$ & $(0.677)$ & $(0.849)$ \\
0.6 & $\mathrm{MC}$ & 0.632 & 0.697 & 0.732 & 0.763 & 0.791 & 0.961 \\
& $\mathrm{AR}$ & $(0.621)$ & $(0.677)$ & $(0.716)$ & $(0.746)$ & $(0.770)$ & $(0.951)$ \\
0.7 & $\mathrm{MC}$ & 0.659 & 0.739 & 0.789 & 0.822 & 0.857 & 0.992 \\
& $\mathrm{AR}$ & $(0.656)$ & $(0.724)$ & $(0.769)$ & $(0.803)$ & $(0.830)$ & $(0.984)$ \\
0.8 & $\mathrm{MC}$ & 0.685 & 0.774 & 0.831 & 0.865 & 0.898 & 0.999 \\
& $\mathrm{AR}$ & $(0.681)$ & $(0.756)$ & $(0.804)$ & $(0.840)$ & $(0.867)$ & $(0.993)$ \\
0.9 & $\mathrm{MC}$ & 0.711 & 0.805 & 0.865 & 0.897 & 0.930 & 1.000 \\
& $\mathrm{AR}$ & $(0.704)$ & $(0.783)$ & $(0.833)$ & $(0.868)$ & $(0.894)$ & $(0.997)$ \\
& & & & & & & \\
1.0 & $\mathrm{MC}$ & 0.731 & 0.831 & 0.892 & 0.927 & 0.950 & 1.000 \\
& $\mathrm{AR}$ & $(0.716)$ & $(0.797)$ & $(0.848)$ & $(0.883)$ & $(0.909)$ & $(0.998)$ \\
1.2 & $\mathrm{MC}$ & 0.757 & 0.863 & 0.920 & 0.955 & 0.970 & 1.000 \\
& $\mathrm{AR}$ & $(0.721)$ & $(0.806)$ & $(0.858)$ & $(0.893)$ & $(0.918)$ & $(0.999)$ \\
& $\mathrm{MC}$ & 0.765 & 0.870 & 0.923 & 0.957 & 0.972 & 1.000 \\
& $\mathrm{AR}$ & $(0.712)$ & $(0.799)$ & $(0.852)$ & $(0.889)$ & $(0.915)$ & $(0.999)$ \\
\hline
\end{tabular}

decreases from 1 for small sample sizes, but for large sample sizes it increases. From the table values, it is observed that in case of log-normal distribution, the PCSs also increase as the shape parameter increases from 0.5 for all sample sizes. When the data are coming from $G E$ distribution, the result is somewhat different. For small samples sizes, as the shape parameter increases from 1, the PCSs do not change much, but for large sample sizes the PCSs increase, but the increase is quite slow. But as the shape parameter decreases from 1, the PCSs increase for all sample sizes. 
Table 3: The probability of correct selection based on Monte Carlo (MC) simulations and also based on asymptotic results (AR) when the data are from $G E$ distribution.

\begin{tabular}{|l|l|l|l|l|l|l|l|}
\hline $\begin{array}{l}n \downarrow \\
\alpha \downarrow\end{array}$ & & 20 & 40 & 60 & 80 & 100 & 500 \\
\hline 0.5 & $\mathrm{MC}$ & 0.637 & 0.733 & 0.760 & 0.801 & 0.828 & 0.971 \\
& $\mathrm{AR}$ & $(0.624)$ & $(0.713)$ & $(0.768)$ & $(0.807)$ & $(0.836)$ & $(0.986)$ \\
0.75 & $\mathrm{MC}$ & 0.520 & 0.580 & 0.614 & 0.667 & 0.672 & 0.790 \\
& $\mathrm{AR}$ & $(0.539)$ & $(0.578)$ & $(0.601)$ & $(0.619)$ & $(0.634)$ & $(0.778)$ \\
1.00 & $\mathrm{MC}$ & 0.360 & 0.433 & 0.462 & 0.479 & 0.485 & 0.507 \\
& $\mathrm{AR}$ & $(0.390)$ & $(0.432)$ & $(0.457)$ & $(0.472)$ & $(0.483)$ & $(0.511)$ \\
1.5 & $\mathrm{MC}$ & 0.274 & 0.405 & 0.491 & 0.507 & 0.556 & 0.816 \\
& $\mathrm{AR}$ & $(0.323)$ & $(0.431)$ & $(0.503)$ & $(0.557)$ & $(0.598)$ & $(0.831)$ \\
2.0 & $\mathrm{MC}$ & 0.271 & 0.420 & 0.513 & 0.563 & 0.620 & 0.934 \\
& $\mathrm{AR}$ & $(0.331)$ & $(0.445)$ & $(0.526)$ & $(0.589)$ & $(0.639)$ & $(0.938)$ \\
2.5 & $\mathrm{MC}$ & 0.273 & 0.429 & 0.524 & 0.591 & 0.650 & 0.969 \\
& $\mathrm{AR}$ & $(0.338)$ & $(0.456)$ & $(0.541)$ & $(0.607)$ & $(0.660)$ & $(0.966)$ \\
& & & & & & & \\
3.0 & $\mathrm{MC}$ & 0.265 & 0.428 & 0.523 & 0.599 & 0.659 & 0.976 \\
& $\mathrm{AR}$ & $(0.341)$ & $(0.460)$ & $(0.545)$ & $(0.612)$ & $(0.665)$ & $(0.967)$ \\
\hline
\end{tabular}

Some of the other interesting findings of these experiments can be obtained when we compare these results with the results when only two distributions have taken at a time. For example, let us compare the PCSs between $G E$ and Weibull (Gupta and Kundu [9]) and GE and log-normal (Kundu, Gupta and Manglick [12]), when the data are from GE distribution and when the shape parameter of the $G E$ distribution is 2.0. Note that when the sample size is 20 and we have only two possibilities namely $G E$ and Weibull then the PCS based on simulation is 0.57 and if the possibilities are $G E$ and log-normal, then the corresponding PCS is 0.68. On the other hand when we have all the three distributions together, then the PCS based on simulation is only 0.271 . Therefore, if the data are coming from $G E$ and even if the 
Table 4: The K-S distances from the $W E(\beta, 1)$, to the closest log-normal $(L N)$ and to the closest $G E$ are presented for different $\beta$.

\begin{tabular}{|l|l|l|l|l|l|l|l|l|}
\hline$\beta \longrightarrow$ & 0.6 & 0.8 & 1.0 & 1.2 & 1.4 & 1.6 & 1.8 & 2.0 \\
\hline$L N$ & 0.071 & 0.071 & 0.071 & 0.071 & 0.071 & 0.071 & 0.071 & 0.071 \\
\hline$G E$ & 0.044 & 0.018 & 0.000 & 0.012 & 0.022 & 0.030 & 0.037 & 0.042 \\
\hline
\end{tabular}

Table 5: The K-S distances from the $L N(\sigma, 1)$, to the closest Weibull $(W E)$ and to the closest $G E$ are presented for different $\sigma$.

\begin{tabular}{|l|l|l|l|l|l|l|l|l|}
\hline$\sigma \longrightarrow$ & 0.5 & 0.6 & 0.7 & 0.8 & 0.9 & 1.0 & 1.2 & 1.4 \\
\hline$W E$ & 0.060 & 0.060 & 0.060 & 0.060 & 0.060 & 0.060 & 0.060 & 0.060 \\
\hline$G E$ & 0.022 & 0.045 & 0.072 & 0.105 & 0.144 & 0.191 & 0.244 & 0.304 \\
\hline
\end{tabular}

shape parameter is quite high, it is difficult to distinguish the three distribution functions. But the same is not true for the Weibull distribution or the log-normal distribution.

These phenomena can be explained using the closest distances between the distribution functions. Let us consider the Kolmogorov-Smirnov (K-S) distances between the three distribution functions. The K-S distance between two distribution functions $F$ and $G$ is defined as follows;

$$
\sup _{-\infty<x<\infty}|F(x)-G(x)|
$$

In the Tables 4, 5 and 6 we present the K-S distances from the parent distribution to the other two closest distribution functions.

Note that when Weibull is the parent distribution then the K-S distance from the Weibull and the closest log-normal is fixed and it does not depend on the shape parameter. But its 
Table 6: The K-S distances from the $G E(\alpha, 1)$, to the closest Weibull( $W E)$ and to the closest log-normal are presented for different $\alpha$.

\begin{tabular}{|l|l|l|l|l|l|l|l|}
\hline$\alpha \longrightarrow$ & 0.5 & 0.75 & 1.0 & 1.5 & 2.0 & 2.5 & 3.0 \\
\hline$W E$ & 0.049 & 0.012 & 0.000 & 0.014 & 0.023 & 0.029 & 0.033 \\
\hline$L N$ & 0.098 & 0.082 & 0.068 & 0.055 & 0.045 & 0.038 & 0.032 \\
\hline
\end{tabular}

$\mathrm{K}-\mathrm{S}$ distance from the Weibull and the closest $G E$ is 0 when both have the shape parameter 1, but the K-S distance gradually increases as the shape parameter moves away from 1 in both direction. When log-normal is the parent distribution, then also as expected the K-S distance between log-normal and the closest Weibull is fixed. But it is observed that the K-S distance between the log-normal and the closest $G E$ gradually decreases as $\sigma$ decreases. Interestingly, when $G E$ is the parent distribution, it has the minimum distance from the Weibull when the shape parameter is one and its distance increases as the shape parameter moves away from one. Although, the K-S distance from the log-normal distribution gradually decreases as the shape parameter increases. Therefore it is clear that for certain range of the $G E$ shape parameter, the three distributions are quite close to each other, and choosing the correct distribution becomes very difficult.

\section{TYPE-I CENSORING}

Since in the reliability analysis most of the times the data are censored, we investigate the effect of Type-I censoring in this discrimination procedure. It was suggested by one of the referees. We use the same maximized likelihood method as in section 2 , and it is assumed that the data are Type-I censored at the time point $y_{c}$. Without loss of generality we take Weibull as the parent distribution. For other two cases, similar results can be obtained. In 
case of censored sample, we denote the differences in ratio of log-likelihoods for the censored sample as $T_{1}^{c}, T_{2}^{c}$ and $T_{3}^{c}$ corresponds to $T_{1}, T_{2}$ and $T_{3}$ respectively, as it was defined for complete sample in section 2. To calculate the PCS theoretically we need the following result.

THEOREM 2: Under the assumptions that the data are from $W E(\beta, \theta),\left(T_{1}^{c}, T_{2}^{c}\right)^{T}$ is asymptotically bivariate normally distributed with mean vector $\left(E_{W E}\left(T_{1}^{c}\right), E_{W E}\left(T_{2}^{c}\right)\right)^{T}$ and dispersion matrix

$$
\Sigma_{W E}^{c}=\left[\begin{array}{cc}
V_{W E}\left(T_{1}^{c}\right) & \operatorname{Cov}_{W E}\left(T_{1}^{c}, T_{2}^{c}\right) \\
\operatorname{Cov}_{W E}\left(T_{1}^{c}, T_{2}^{c}\right) & V_{W E}\left(T_{2}^{c}\right)
\end{array}\right]
$$

Proof : The proof of this theorem can be carried out exactly like that of complete sample case.

We have reported results for three different $y_{c}$, namely at 90-th, 80-th and 70-th percentile points of the respective parent distributions.

Table 7: The Probability of Correct Selection based on Monte Carlo simulations (MC) and also based on asymptotic results (AR), when the parent distribution is $\mathrm{WE}(2,1)$.

\begin{tabular}{|l|l|l|l|l|l|}
\hline$p$ & $y_{c}$ & & $n=40$ & $n=60$ & $n=80$ \\
\hline 0.7 & 1.10 & MC & 0.548 & 0.599 & 0.640 \\
& & AR & $(0.624)$ & $(0.659)$ & $(0.687)$ \\
\hline 0.8 & 1.27 & MC & 0.596 & 0.658 & 0.698 \\
& & AR & $(0.656)$ & $(0.696)$ & $(0.728)$ \\
\hline 0.9 & 1.52 & MC & 0.659 & 0.718 & 0.759 \\
& & AR & $(0.695)$ & $(0.742)$ & $(0.777)$ \\
\hline
\end{tabular}

In all the three cases as expected, it is observed that as $y_{c}$ decreases the probability of correct selection decreases. Moreover comparing the results with the complete sample results it is also observed that discrimination becomes more difficult (PCS is lower) when the data are censored compared to the complete sample case as the intuition suggests. 
Table 8: The Probability of Correct Selection based on Monte Carlo simulations (MC) and also based on asymptotic results (AR), when the parent distribution is $\operatorname{LN}(2,1)$

\begin{tabular}{|l|l|l|l|l|l|}
\hline$p$ & $y_{c}$ & & $n=40$ & $n=60$ & $n=80$ \\
\hline 0.7 & 2.85 & MC & 0.831 & 0.857 & 0.874 \\
& & AR & $(0.781)$ & $(0.838)$ & $(0.874)$ \\
\hline 0.8 & 5.38 & MC & 0.854 & 0.886 & 0.910 \\
& & AR & $(0.830)$ & $(0.879)$ & $(0.912)$ \\
\hline 0.9 & \multirow{2}{*}{12.98} & MC & 0.869 & 0.909 & 0.935 \\
& & AR & $(0.861)$ & $(0.913)$ & $(0.943)$ \\
\hline
\end{tabular}

Table 9: The Probability of Correct Selection based on Monte Carlo simulations when the parent distribution is $G E(2,1)$.

\begin{tabular}{|l|l|l|l|l|}
\hline$p$ & $y_{c}$ & $n=40$ & $n=60$ & $n=80$ \\
\hline 0.7 & 1.81 & 0.256 & 0.335 & 0.388 \\
& & 0.271 & 0.324 & 0.392 \\
\hline 0.8 & 2.24 & 0.307 & 0.392 & 0.445 \\
& & 0.329 & 0.392 & 0.443 \\
\hline 0.9 & 2.97 & 0.350 & 0.446 & 0.506 \\
& & 0.375 & 0.447 & 0.504 \\
\hline
\end{tabular}

\section{Data Analysis}

In this section we analyze two real data sets for illustrative purposes. In both the cases the sample size is not very large. It is observed that for one case the choice is quite clear but for the other case it is not very easy to make a decision about the model choice.

DATA SET 1: The first data set (Linhardt and Zucchini [16], page 69); represents the failure times of the air conditioning system of an airplane and it is as follows: $23,261,87,7,120$, 14, 62, 47, 225, 71, 246, 21, 42, 20, 5, 12, 120, 11, 3, 14, 71, 11, 14, 11, 16, 90, 1, 16, 52, 95.

We obtain the following estimates of different model parameters.

- Weibull distribution: $\widehat{\beta}=0.8535, \quad \widehat{\theta}=0.0183, \quad \ln L_{W E}(\widehat{\beta}, \widehat{\theta})=-151.937$. 
- Log-normal distribution: $\widehat{\sigma}=1.3192, \quad \widehat{\eta}=28.7343, \quad \ln L_{L N}(\widehat{\sigma}, \widehat{\eta})=-151.621$.

- $G E$ distribution: $\widehat{\alpha}=0.8092, \quad \widehat{\lambda}=0.0145, \quad \ln L_{G E}(\widehat{\alpha}, \widehat{\lambda})=-152.201$.

Therefore, based on the log-likelihood values log-normal is the preferred model. We also computed the Kolmogorov-Smirnov (K-S) distances between the empirical distribution function (EDF) and the fitted models in each case and they are 0.1047, 0.1540 and 0.1744 from the log-normal, Weibull and GE and the corresponding $p$-values are $0.88,0.44$ and 0.32 respectively. We have also used re-sampling technique (non-parametric Bootstrap) to compute the proportion that it chooses different distributions. It is observed that after re sampling with replacement it chooses log-normal, Weibull and GE at 44.4\%, 39.0\% and $16.6 \%$ respectively. Therefore, based on all these information we will choose log-normal as the preferred model in this case.

DATA SET 2: The data represents the number of revolution (million) before failure of the 23 ball bearings in the life-test and it was originally reported in Lawless [15] (page 228). It is as follows: $17.88,28.92,33.00,41.52,42.12,45.60,48.80,51.84,51.96,54.12,55.56,67.80$, 68.44, 68.64, 68.88. 84.12, 93.12, 98.64, 105.12, 105.84, 127.92, 128.04, 173.40. Using the three different models, we obtain the following:

- Weibull distribution: $\widehat{\beta}=2.1036, \quad \widehat{\theta}=0.0122, \quad \ln L_{W E}(\widehat{\beta}, \widehat{\theta})=-113.689$.

- Log-normal distribution: $\widehat{\sigma}=0.5214, \quad \widehat{\eta}=63.4729, \quad \ln L_{L N}(\widehat{\sigma}, \widehat{\eta})=-113.125$.

- $G E$ distribution: $\widehat{\alpha}=5.2838, \quad \widehat{\lambda}=0.0323, \quad \ln L_{G E}(\widehat{\alpha}, \widehat{\lambda})=-112.973$.

Therefore, based on the log-likelihood criterion, it is observed that we prefer the $G E$ distribution. The K-S distances from the EDF to the fitted log-normal, Weibull and GE are 0.0901, 0.1521 and 0.1262 and the corresponding $p$ values are $0.98,0.63$ and 0.86 respectively. 
Using re-sampling technique it is observed that it chooses the log-normal, Weibull and GE as $47 \%, 32 \%$ and $21 \%$ respectively. Therefore, it shows that although log-likelihood prefers to choose GE but other criteria do not prefer GE. From these two examples and also from the simulation results, it is clear that not only the sample sizes, but also the model parameters play important roles in discriminating between closely fitted models.

\section{Determination of Sample Size}

In this section, we propose a method to determine the minimum sample size needed to discriminate among the three distribution functions for a given user specified probability of correct selection. Intuitively, if the distances among several distributions are large, one may not need very large sample to discriminate them. On the other hand if they are very close to each other it will be extremely difficult to discriminate them and it may not be necessary from any practical point of view. Therefore, it is expected that user will specify before-hand the PCS and also the tolerance limit (similar to type-I error) in terms of the minimum distances (tolerance level) among several distribution functions, for discrimination purposes, i.e., we do not want to discriminate between two distribution functions if the distance between them is less than the tolerance level, see Gupta and Kundu [9] in this respect. Here we use the K-S distance as a measure of distance between two distributions, similarly as in Gupta and Kundu [9].

We have already reported the K-S distances between any two distribution functions in Tables 4, 5 and 6 . It is interesting to observe that the K-S distances between log-normal and Weibull are constant for all parameter ranges. The K-S distance between Weibull and generalized exponential distributions is minimum when the shape parameter is 1 (both are exponential) and it gradually increases as the shape parameter moves away from 1 . The K-S distance between log-normal and generalized exponential distributions decreases as the 
shape parameter of the generalized exponential distribution increases or the shape parameter of the log-normal distribution decreases.

Now suppose we want to compute the minimum sample size needed to achieve the probability of correct selection to be 0.65 and when the tolerance limit is 0.03 , i.e. we do not want to discriminate among the distributions if their K-S distances are less than 0.03. Now if Weibull is the parent distribution, it is clear from the Table 4 that if $\beta \geq 1.6$ or $\beta \leq 0.7$ (approximately), then the K-S distances among the three distribution functions will be more than the tolerance level. Similarly, if log-normal is the parent distribution then for $\sigma \geq 0.55$ (approximately) and if generalized exponential distribution is the parent distribution then for $\alpha \leq 0.55$ (approximately) or $2.5<\alpha<3.25$ (approximately), this criterion will be met. Now if Weibull is the parent distribution, from Table 1 it is clear that we need at least a sample of size 60 to achieve this probability of correct selection. From Table 2, it is observed that if log-normal is the parent distribution then we need at least sample size 40 (approximately) to achieve this probability of correct selection. Similarly, when the generalized exponential distribution is the parent distribution, then from Table 3 we observe that we need at least a sample of size 100 to achieve the required probability of correct selection. Therefore, at

least $\max \{60,40,100\}=100$ sample size is required to achieve the required probability of correct selection 0.65 with the tolerance level 0.03 . Similarly it can be obtained for other cases also.

\section{Conclusions}

In this paper we have considered the problem of discriminating among three important lifetime distribution functions. We have used the maximized likelihood method to choose the best fitted model. We have obtained the asymptotic distributions of the ratio of the maximized log-likelihood functions and it is observed that it follows bivariate normal distribution. 
Based on the asymptotic results we have computed the probability of correct selections and they have been compared with the simulated results. The results have been extended for the Type-I censored data also. It is observed that they match quite well even for moderate sample sizes. It is also observed that the probability of correct selection not only depends on the sample sizes, but it also depends on the model parameters of the parent population. We have also suggested how to obtain the minimum sample size required to achieve a given probability of correct selection for a user specified protection level. Although we have considered only Weibull, log-normal and generalized exponential distributions, but our method can be extended for other distribution functions also.

\section{ACKNOWLEDGEMENTS}

The authors would like to thank three reviewers for several constructive suggestions and also to Professor Lesley Walls for encouragements.

\section{APPENDIX - A}

In this section we present the exact expressions of the mean vectors and the elements of the dispersion matrices for the three different cases. The exact expressions of most of the elements are already available in the literature. Whichever are not available we will present those explicitly, otherwise we will provide the references.

\section{Parent Distribution is Weibull}

First we need the exact expressions $\sigma_{1}, \eta_{1}, \alpha_{1}$ and $\lambda_{1}$ of Lemma 1 , as all the required elements are functions of these. Note that

$$
\sigma_{1}=\frac{\sqrt{\psi^{\prime}(1)}}{\beta}, \quad \eta_{1}=\frac{1}{\theta} e^{\frac{\psi(1)}{\beta}},
$$


see for details Kundu and Manglick [13]. Here $\psi(x)=\frac{d}{d x} \log \Gamma(x)$ and $\psi^{\prime}(x)$ is the derivative of $\psi(x)$. Although, $\sigma_{1}, \eta_{1}$ have explicit forms in terms of $\beta$, but $\alpha_{1}$ and $\lambda_{1}$ do not have explicit forms. They can be obtained as the roots of the two non-linear equations, see Gupta and Kundu [9] for details and also tabulated values of $\alpha_{1}$ and $\lambda_{1}$ for different values of $\beta$. The exact expressions of

$$
\lim _{n \longrightarrow \infty} \frac{1}{n} E_{W E}\left(T_{1}\right) \quad \text { and } \lim _{n \longrightarrow \infty} \frac{1}{n} V_{W E}\left(T_{1}\right)
$$

are available in Kundu and Manglick [13], similarly, the exact expressions of

$$
\lim _{n \longrightarrow \infty} \frac{1}{n} E_{W E}\left(T_{2}\right) \quad \text { and } \lim _{n \longrightarrow \infty} \frac{1}{n} V_{W E}\left(T_{2}\right)
$$

are available in Gupta and Kundu [9]. Now we present the $\operatorname{Cov}_{W E}\left(T_{1}, T_{2}\right)$, which is not available anywhere. Note that if $Z$ follows $W E(\beta, 1)$, then

$$
\begin{aligned}
\lim _{n \rightarrow \infty} \frac{1}{n} \operatorname{Cov}_{W E}\left(T_{1}, T_{2}\right) & =\operatorname{Cov}_{W E}\left[\ln \frac{f_{W E}(X ; \beta, \theta)}{f_{L N}\left(X ; \sigma_{1}, \eta_{1}\right)}, \ln \frac{f_{W E}(X ; \beta, \theta)}{f_{G E}\left(X ; \alpha_{1}, \lambda_{1}\right)}\right] \\
& =(\beta-1)\left(\beta-\frac{\ln \eta_{1}}{\sigma_{1}^{2}}\right) \frac{\psi^{\prime}(1)}{\beta^{2}}-(\beta-1) \frac{\left(\psi^{\prime}(2)-\psi^{\prime}(1)\right)}{\beta} \\
& +\frac{1}{2 \sigma_{1}^{2}}(\beta-1)\left(\psi^{\prime \prime}(1)-2(\psi(1))^{3}\right)-\left(\beta-\frac{\ln \eta_{1}}{\sigma_{1}^{2}}\right) \frac{(\psi(2)-\psi(1))}{\beta} \\
& +1-\frac{1}{2 \sigma_{1}^{2}}\left(\frac{\left(\psi^{\prime}(2)-\psi^{\prime}(1)\right)}{\beta^{2}}+\frac{\psi^{2}(2)-\psi^{2}(1)}{\beta^{2}}\right) \\
& -(\alpha-1)\left(\beta-\frac{\ln \eta_{1}}{\sigma_{1}^{2}}\right) C_{o v}\left(\ln Z, \ln \left(1-\exp \left(-\lambda_{1} Z\right)\right)\right) \\
& +(\alpha-1) C o v_{W E}\left(\ln \left(1-\exp \left(-\lambda_{1} Z\right)\right), Z^{\beta}\right) \\
& -(\alpha-1) \frac{1}{2 \sigma_{1}^{2}} C_{o v}\left(\ln \left(1-\exp \left(-\lambda_{1} z\right)\right),(\ln Z)^{2}\right) \\
& +\lambda_{1}\left(\beta-\frac{\ln \eta_{1}}{\sigma_{1}^{2}}\right) \frac{1}{\beta}\left[\psi\left(\frac{1}{\beta}+1\right) \Gamma\left(\frac{1}{\beta}+1\right)-\Gamma\left(\frac{1}{\beta}+1\right) \psi(1)\right] \\
& -\lambda_{1}\left[\Gamma\left(\frac{\beta+1}{\beta}+1\right)-\Gamma\left(\frac{1}{\beta}+1\right)\right] \\
& +\frac{\lambda_{1}}{2 \sigma_{1}^{2}}\left(\Gamma\left(\frac{1}{\beta}+1\right) \frac{\left[\psi^{\prime}\left(\frac{1}{\beta}+1\right)+\psi^{2}\left(\frac{1}{\beta}+1\right)\right]}{\beta^{2}}-\frac{\Gamma\left(\frac{1}{\beta}+1\right)}{\beta^{2}}\left(\psi^{\prime}(1)+\psi^{2}(1)\right)\right) .
\end{aligned}
$$

PARent Distribution is Log-Normal 
Note that when the data are from $L N(\sigma, \eta)$, then along the same line as Lemma 1, it can be shown

$$
\widehat{\beta} \longrightarrow \beta_{2} \text {, a.s. } \widehat{\theta} \longrightarrow \theta_{2} \text { a.s. } \widehat{\alpha} \longrightarrow \alpha_{2} \text { a.s. } \widehat{\lambda} \longrightarrow \lambda_{2} \text { a.s. }
$$

where $\beta_{2}=\frac{1}{\sigma}$ and $\theta_{2}=\frac{1}{\eta} e^{-\frac{\sigma}{2}}$, see Kundu and Manglick [13]. The exact expressions for $\alpha_{2}$ and $\lambda_{2}$ are not possible to obtain. They can be obtained by solving two non-linear equations, see Kundu, Gupta and Manglick [12]. The exact expressions of

$$
\lim _{n \longrightarrow \infty} \frac{1}{n} E_{L N}\left(T_{3}\right) \quad \text { and } \lim _{n \longrightarrow \infty} \frac{1}{n} V_{L N}\left(T_{3}\right)
$$

are available in Kundu, Gupta and Manglick [12], similarly, the exact expressions of

$$
\lim _{n \longrightarrow \infty} \frac{1}{n} E_{L N}\left(T_{1}\right) \quad \text { and } \lim _{n \longrightarrow \infty} \frac{1}{n} V_{L N}\left(T_{1}\right)
$$

are available in Kundu and Manglick [13]. Now we present the $\operatorname{Cov}_{L N}\left(T_{3}, T_{1}\right)$, which is not available anywhere. Note that if $Z$ follows $L N(\sigma, 1)$, then

$$
\begin{aligned}
\lim _{n \rightarrow \infty} \frac{1}{n} \operatorname{Cov}_{L N}\left(T_{3}, T_{1}\right) & =\operatorname{Cov}_{L N}\left[\ln \frac{f_{L N}(X ; \sigma, \eta)}{f_{G E}\left(X ; \alpha_{2}, \lambda_{2}\right)},-\ln \frac{f_{L N}(X ; \sigma, \eta)}{f_{W E}\left(X ; \beta_{2}, \theta_{2}\right)}\right] \\
& =-2 \beta_{2} \sigma^{4}+\lambda_{2} \beta_{2} \sigma^{2} e^{\frac{\sigma^{2}}{2}} \\
& -\beta_{2}\left(\alpha_{2}-1\right) \operatorname{Cov}_{L N}\left(\ln Z, \ln \left(1-\exp \left(-\lambda_{2} Z\right)\right)\right) \\
& +\frac{1}{2} \lambda_{2} \sigma^{2} e^{\frac{\sigma^{2}}{2}}-\frac{1}{2} \sigma^{2}-\frac{\alpha_{2}-1}{2 \sigma^{2}} \operatorname{Cov}_{L N}\left((\ln Z)^{2}, \ln \left(1-\exp \left(-\lambda_{2} Z\right)\right)\right) \\
& +\theta_{2}^{\beta_{2}} \operatorname{Cov}_{L N}\left(Z^{\beta_{2}},(\ln Z)^{2}\right)+\left(\alpha_{2}-1\right) \theta^{\beta_{2}} \operatorname{Cov}_{L N}\left(Z^{\beta_{2}}, \ln \left(1-\exp \left(-\lambda_{2} Z\right)\right)\right)
\end{aligned}
$$

\section{Parent Distribution is Generalized Exponential}

When the data are from $G E(\alpha, \lambda)$, then it follows that

$$
\widehat{\beta} \longrightarrow \beta_{3}, \quad \text { a.s., } \quad \hat{\theta} \longrightarrow \theta_{3} \quad \text { a.s. } \quad \widehat{\sigma} \longrightarrow \sigma_{3}, \quad \text { a.s. } \quad \widehat{\eta} \longrightarrow \eta_{3} \text { a.s. }
$$

The expressions for $\beta_{3}, \theta_{3}, \lim _{n \longrightarrow \infty} \frac{1}{n} E_{G E}\left(T_{2}\right), \lim _{n \longrightarrow \infty} \frac{1}{n} V_{G E}\left(T_{2}\right)$ are available in Gupta and Kundu [9]. Moreover, the expressions for $\sigma_{3}, \eta_{3}, \lim _{n \longrightarrow \infty} \frac{1}{n} E_{G E}\left(T_{3}\right), \lim _{n \longrightarrow \infty} \frac{1}{n} V_{G E}\left(T_{3}\right)$ are available in Kundu, Gupta and Manglick [12]. If $Z$ follows $G E(\alpha, \lambda)$, then $\operatorname{Cov}_{G E}\left(T_{2}, T_{3}\right)$, can be 
written as

$$
\begin{aligned}
\lim _{n \longrightarrow \infty} \frac{1}{n} \operatorname{Cov}_{G E}\left(T_{3}, T_{2}\right) & =\operatorname{Cov}_{G E}\left[-\ln \frac{f_{G E}(X ; \alpha, \lambda)}{f_{W E}\left(X ; \beta_{3}, \theta_{3}\right)},-\ln \frac{f_{G E}(X ; \alpha, \lambda)}{f_{L N}\left(X ; \sigma_{3}, \eta_{3}\right)}\right] \\
& =\left(\frac{\alpha-1}{\alpha}\right)^{2}+\left(\psi^{\prime}(1)-\psi^{\prime}(\alpha+1)\right)-2(\alpha-1) \operatorname{Cov}_{G E}(\ln (1-\exp (-z)), z) \\
& +\frac{\alpha-1}{2 \sigma_{3}^{2}} \operatorname{Cov}_{G E}\left(\ln (1-\exp (-Z)),(\ln Z)^{2}\right) \\
& +(\alpha-1)\left(1-\frac{\theta_{3}}{\sigma_{3}^{2}}\right) \operatorname{Cov}_{G E}(\ln Z, \ln (1-\exp (-Z))) \\
& -\frac{1}{2 \sigma_{3}^{2}} \operatorname{Cov}_{G E}\left(Z,(\ln Z)^{2}\right)-\left(1-\frac{\ln \theta_{3}}{\sigma_{3}^{2}}\right) \operatorname{Cov}_{G E}(Z, \ln Z) \\
& -(\alpha-1)\left(\beta_{3}-1\right) \operatorname{Cov}_{G E}(\ln Z, \ln (1-\exp (-Z)))+\left(\beta_{3}-1\right) \operatorname{Cov}_{G E}(Z, \ln Z) \\
& -\frac{\beta_{3}-1}{2 \sigma_{3}^{2}} \operatorname{Cov}_{G E}\left(\ln Z,(\ln Z)^{2}\right)-\left(\beta_{3}-1\right)\left(1-\frac{\ln \theta_{3}}{\sigma_{3}^{2}}\right) V_{G E}(\ln Z) \\
& +(\alpha-1) \theta_{3}^{\beta_{3}} \operatorname{Cov}_{G E}\left(\ln (1-\exp (-Z)), Z^{\beta_{3}}\right) \\
& -\theta_{3}^{\beta_{3}} \operatorname{Cov}_{G E}\left(Z, Z^{\beta_{3}}\right)+\theta_{3}^{\beta_{3}} \frac{1}{2 \sigma_{3}^{2}} \operatorname{Cov}_{G E}\left(Z^{\beta_{3}},(\ln Z)^{2}\right) \\
& +\theta_{3}^{\beta_{3}}\left(1-\frac{\ln \theta_{3}}{\sigma_{3}^{2}}\right) \operatorname{Cov}_{G E}\left(\ln Z, Z^{\beta_{3}}\right) .
\end{aligned}
$$

\section{Appendix - B}

Suppose, the parent distribution is Weibull and the data are Type-I censored.

Let us define, $a_{1}=\ln \beta+\beta \ln \theta-\ln \alpha-\ln \lambda, a_{2}=\ln \beta+\beta \ln \theta+0.5 \ln (2 \pi)+\ln \sigma$,

$$
\begin{aligned}
T_{1}^{* c}=\sum_{i=1}^{n}\left\{\Delta_{i}\left[\ln f_{W E}\left(X_{i} ; \beta, \theta\right)-\ln f_{L N}\left(X_{i} ; \sigma_{1}, \eta_{1}\right)\right]+\right. \\
\left.\left(1-\Delta_{i}\right)\left[\ln \left(\bar{F}_{W E}\left(y_{c} ; \beta, \theta\right)\right)-\ln \left(\bar{F}_{L N}\left(y_{c} ; \sigma_{1}, \eta_{1}\right)\right)\right]\right\}, \\
T_{2}^{* c}=\sum_{i=1}^{n}\left\{\Delta_{i}\left[\ln f_{W E}\left(X_{i} ; \beta, \theta\right)-\ln f_{G E}\left(X_{i} ; \alpha_{1}, \lambda_{1}\right)\right]+\right. \\
\left.\left(1-\Delta_{i}\right)\left[\ln \left(\bar{F}_{W E}\left(y_{c} ; \beta, \theta\right)\right)-\ln \left(\bar{F}_{G E}\left(y_{c} ; \alpha_{1}, \lambda_{1}\right)\right)\right]\right\},
\end{aligned}
$$

where $\bar{F}=(1-F)$ and

$$
\Delta_{i}=\left\{\begin{array}{cl}
1 & \text { if } X_{i} \leq y_{c} \\
0 & \text { if } X_{i}>y_{c} .
\end{array}\right.
$$


Here $X_{i}$ is the $i$-th observation. Therefore the expression for expectations, variances and covariance can be given by

$$
\begin{aligned}
& \lim _{n \longrightarrow \infty} \frac{E_{W E}\left(T_{1}^{c}\right)}{n}=E_{W E}\left\{\left(\Delta_{1}\left[\ln f_{W E}\left(X_{1} ; \beta, \theta\right)-\ln f_{L N}\left(X_{1} ; \sigma_{1}, \eta_{1}\right)\right]+\right.\right. \\
& \left.\left.\left(1-\Delta_{1}\right)\left[\ln \left(\bar{F}_{W E}\left(y_{c} ; \beta, \theta\right)\right)-\ln \left(\bar{F}_{L N}\left(y_{c} ; \sigma_{1}, \eta_{1}\right)\right)\right]\right)\right\} \text {. } \\
& \lim _{n \longrightarrow \infty} \frac{E_{W E}\left(T_{2}^{c}\right)}{n}=E_{W E}\left\{\left(\Delta_{i}\left[\ln f_{W E}\left(X_{i} ; \beta, \theta\right)-\ln f_{G E}\left(X_{i} ; \alpha_{1}, \lambda_{1}\right)\right]+\right.\right. \\
& \left.\left.\left(1-\Delta_{i}\right)\left[\ln \left(\bar{F}_{W E}\left(y_{c} ; \beta, \theta\right)\right)-\ln \left(\bar{F}_{G E}\left(y_{c} ; \alpha_{1}, \lambda_{1}\right)\right)\right]\right)\right\} \text {. } \\
& \lim _{n \longrightarrow \infty} \frac{V_{W E}\left(T_{1}^{c}\right)}{n}=V_{W E}\left\{\left(\Delta_{1}\left[\ln f_{W E}\left(X_{1} ; \beta, \theta\right)-\ln f_{L N}\left(X_{1} ; \sigma_{1}, \eta_{1}\right)\right]+\right.\right. \\
& \left.\left.\left(1-\Delta_{1}\right)\left[\ln \left(\bar{F}_{W E}\left(y_{c} ; \beta, \theta\right)\right)-\ln \left(\bar{F}_{L N}\left(y_{c} ; \sigma_{1}, \eta_{1}\right)\right)\right]\right)\right\} \text {. } \\
& \lim _{n \longrightarrow \infty} \frac{V_{W E}\left(T_{2}^{c}\right)}{n}=V_{W E}\left\{\left(\Delta_{1}\left[\ln f_{W E}\left(X_{1} ; \beta, \theta\right)-\ln f_{G E}\left(X_{1} ; \alpha_{1}, \lambda_{1}\right)\right]+\right.\right. \\
& \left.\left.\left(1-\Delta_{1}\right)\left[\ln \left(\bar{F}_{W E}\left(y_{c} ; \beta, \theta\right)\right)-\ln \left(\bar{F}_{G E}\left(y_{c} ; \alpha_{1}, \lambda_{1}\right)\right)\right]\right)\right\} \text {. } \\
& \begin{aligned}
\lim _{n \longrightarrow \infty} \frac{\operatorname{Cov}_{W E}\left\{T_{1}^{c}, T_{2}^{c}\right\}}{n}=\operatorname{Cov}_{W E}\left\{\Delta_{1}\left[\ln f_{W E}\left(X_{1} ; \beta, \theta\right)-\ln f_{L N}\left(X_{1} ; \sigma_{1}, \eta_{1}\right)\right]+\right. \\
\left(1-\Delta_{1}\right)\left[\ln \left(\bar{F}_{W E}\left(y_{c} ; \beta, \theta\right)\right)-\ln \left(\bar{F}_{L N}\left(y_{c} ; \sigma_{1}, \eta_{1}\right)\right)\right] \\
\Delta_{1}\left[\ln f_{W E}\left(X_{1} ; \beta, \theta\right)-\ln f_{G E}\left(X_{1} ; \alpha_{1}, \lambda_{1}\right)\right]+ \\
\left.\left(1-\Delta_{1}\right)\left[\ln \left(\bar{F}_{W E}\left(y_{c} ; \beta, \theta\right)\right)-\ln \left(\bar{F}_{G E}\left(y_{c} ; \alpha_{1}, \lambda_{1}\right)\right)\right]\right\}
\end{aligned}
\end{aligned}
$$

Other expressions can be given in the similar fashion.

\section{References}

[1] Atkinson, A. (1970), "A method for discriminating between models" (with discussions), Journal of the Royal Statistical Society, Ser. B, vol. 32, 323-353. 
[2] Bain, L.J. and Englehardt, M. (1980), "Probability of correct selection of Weibull versus gamma based on likelihood ratio", Communications in Statistics, Ser. A., vol. $9,375-381$.

[3] Chambers, E.A. and Cox, D.R. (1967), "Discriminating between alternative binary response models", Biometrika, 54, 573-578.

[4] Chen, W.W. (1980), "On the tests of separate families of hypotheses with small sample size", Journal of Statistical Computations and Simulations, vol. 2, 183-187.

[5] Cox, D.R. (1961), "Tests of separate families of hypotheses", Proceedings of the Fourth Berkeley Symposium in Mathematical Statistics and Probability, Berkeley, University of California Press, 105-123.

[6] Cox, D.R. (1962), "Further results on tests of separate families of hypotheses", Journal of the Royal Statistical Society, Ser. B, vol. 24, 406-424.

[7] Dumonceaux, R. and Antle, C.E. (1973), "Discriminating between the log-normal and Weibull distribution", Technometrics, vol. 15, 923-926.

[8] Fearn, D.H. and Nebenzahl, E. (1991), "On the maximum likelihood ratio method of deciding between the Weibull and Gamma distributions", Communications in Statistics - Theory and Methods, vol. 20, 579-593.

[9] Gupta, R. D. and Kundu, D. (2003a), "Discriminating between the Weibull and the GE distributions", Computational Statistics and Data Analysis, vol. 43, 179 196.

[10] Kappenman, R.F. (1982), "On a method for selecting a distributional model", Communications in Statistics - Theory and Methods, vol.11, 663-672. 
[11] Keats, J. B., Nahar, P.C. and Korbell, K.M. (2000), "A study of the effect of misspecification of the Weibull shape parameter on confidence bounds based on the Weibull-exponential transformation", Quality and Reliability Engineering International, vol. $16,27-31$.

[12] Kundu, D., Gupta, R.D. and Manglick, A. (2005), "Discriminating between the lognormal and generalized exponential distributions", Journal of Statistical Planning and Inference, vol. 127, 213 - 227.

[13] Kundu, D. and Manglick, A. (2004), "Discriminating between the Weibull and Log-Normal distributions", Naval Research Logistics, vol. 51, 893-905, 2004.

[14] Kundu, D. and Manglick, A. (2005), "Discriminating between the Log-Normal and gamma distributions", Journal of the Applied Statistical Sciences, vol. 14, 175-187, 2005 .

[15] Lawless, J.F. (1982), Statistical Models and Methods for Lifetime Data, John Wiley and Sons, New York.

[16] Linhardt, H. and Zucchini, W. (1986), Model Selection, Wiley, New York.

[17] Marshall, A.W., Meza, J.C. and Olkin, I. (2001), "Can data recognize its parent distribution?", Journal of Computational and Graphical Statistics, vol. 10, 555 580.

[18] Pascual, F.G. (2005), "Maximum likelihood estimation under misspecified lognormal and Weibull distributions", Communications in Statistics - Simulation and Computations vol. 34, 503 - 524, 2005.

[19] Pereira, B, de (1978), "Empirical comparison of some tests of separate families of hypothesis", Metrika, vol. 25, 219 - 234. 
[20] Press, W.H., Flannery, B.P., Teukolsky, S.A. and Vetterling, W.T. (1993), Numerical Recipes; The Art of Scientific Computing, Cambridge University Press, Cambridge, U.K.

[21] Schneider, H. (1989), "Failure-censored variables-sampling plans for log-normal and Weibull distributions", Technometrics, vol. 31, no. 2, 199 - 206.

[22] Taylor, J.A. and Jakeman, A.J. (1985), "Identification of a distributional model", Communications in Statistics - Theory and Methods, vol. 14, 497-508.

[23] Quesenberry, C.P. and Kent, J. (1982), "Selecting among probability distributions used in reliability", Technometrics, vol. 24, 59-65.

[24] White, H. (1982a), "Maximum likelihood estimation of mis-specified models", Econometrica, vol. 50, 1-25.

[25] White, H. (1982b), "Regularity conditions for Cox's test of non-nested hypotheses", Journal of Econometrics, vol. 19, 301-318.

[26] Wiens, B.L. (1999), "When log-normal and gamma models give different results: a case study", American Statistician, vol. 53, 89-93.

[27] Xie, M., Yang, Z. and Gaudoin, O. (2000), "More on the mis-specification of the shape parameter with Weibull to exponential transformation", Quality and Reliability Engineering International, vol. 16, 281 - 290. 